\title{
Expansion of capillary force range by probe-tip curvature
}

\author{
K. J. Obata ${ }^{1}$, S. Saito ${ }^{2} \&$ K. Takahashi ${ }^{1}$ \\ ${ }^{1}$ Department of International Development Engineering, \\ Tokyo Institute of Technology, Japan \\ ${ }^{2}$ Department of Mechanical and Aerospace Engineering, \\ Tokyo Institute of Technology, Japan
}

\begin{abstract}
This paper deals with the expansion of capillary force range with a concave probetip for micromanipulation. From numerical simulation, we found the following; the concave probe-tip can generate a much larger capillary than a flat one, provided it is designed to fit the convex surface of the object; the more wettable a material is, the greater capillary force it can generate; the magnified capillary force can be reduced/controlled by liquid volume regulation. To prove this, we measured the capillary force for a given gap distance between a spherical object and a concave surface coaxially fabricated in a cylinder. We used three different materials (glass, stainless steel, and polytetrafluoroethylene) to check the influence of contact angles. The liquid volumes were given in the range from one hundredth- to ten-times the radius of the cubed sphere. Comparison between our experimental data and the theoretical prediction expressed in the normalized form shows good agreement, if the liquid volume is larger than a certain value. This suggests that micromanipulation by capillary force should be more practical by using probes with concave tips specifically designed for the object.

Keywords: micromanipulation, hydrostatics, liquid bridge, capillary force.
\end{abstract}

\section{Introduction}

Recently, micromanipulation techniques have been in demand to fabricate highly functional micro-devices or micro-electro-mechanical-systems (MEMS). In micromanipulation, the influence of adhesional force is extremely large compared to gravitational force $[1,2]$. Furthermore, adhesional force has large dispersion 
because of its dependence on surface condition such as surface roughness, at each contact point. Thus, in order to realize reliable micromanipulation, we need a force that is controllable and greater than the adhesional force, or some mechanism to avoid the dispersion of adhesional force. Saito et al $[3,4]$ have investigated the mechanical force required to slip and roll an object in considering the adhesional effect. The mechanical method, however, might damage the object. Takahashi et al [5] have evaluated the force generated by Coulomb interaction, although the electrostatic method might cause a discharge or melt an object [6]. In order to avoid damaging the manipulated object, use of capillary force is considered effective if use of liquid is allowed. Actually, Tanikawa et al [7] have picked/placed an object with a micro-hand and a micro-drop, but they have not provided any analysis of the capillary force involved. We have proposed a scheme for micromanipulation based on capillary force by regulation of the liquid volume [8]. In our previous scheme, it is assumed that the object shape is spherical, and the probe and substrate surfaces are flat. The profile of a liquid bridge between two solids was analyzed based on Orr's theory [9], and the force generated for the profile was presented clearly. These analyses have indicated the feasibility of the proposed scheme. The range of the force, however, never seems large enough for practical/reliable micromanipulation. In this study, we propose a probe with a concave-tip as shown in fig. 1. The concaved probe-tip would generate greater capillary force than a flat probe-tip so that it can expand the possibility of picking up manipulation. If a large amount of liquid was supplied, liquid must overflow to a flat surface, and capillary force would be equivalent to the flat probe-tip case for successful placing. Through both numerical estimation and its experimental verification, the magnified range of capillary force is presented as a function of concavity radius, wettability, and liquid volume.

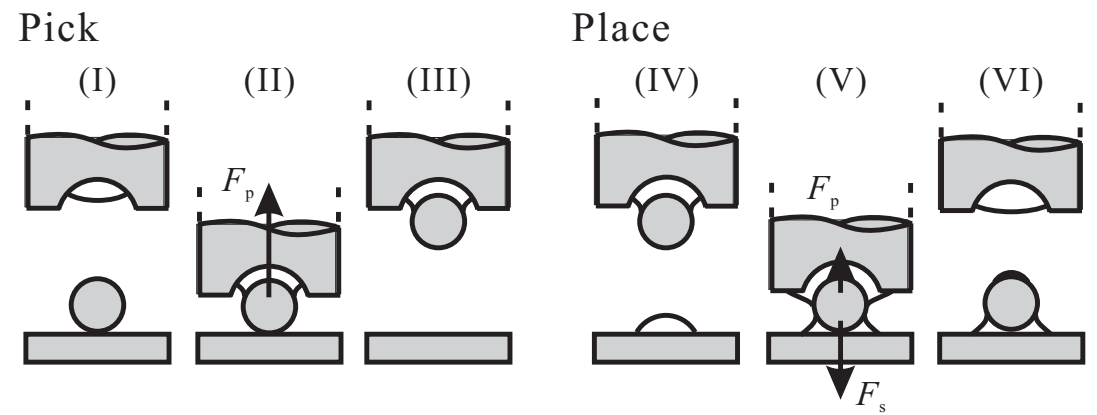

Figure 1: Schematic illustration of manipulation procedure: (I) positioning, (II) lowering, (III) picking up, (IV) positioning, (V) lowering, and (VI) placing. 
(a)

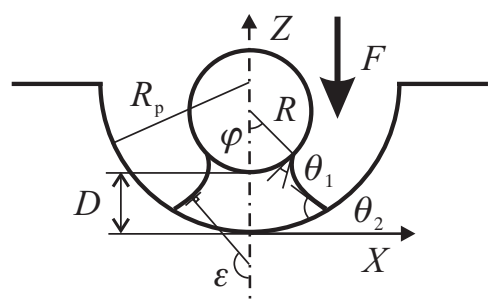

(b)

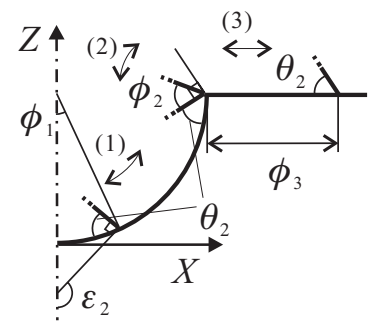

Figure 2: Liquid bridge between a spherical object and a concave shaped probe: (a) whole menisci (b) menisci end at the probe surface.

\section{Analysis of the liquid bridge}

Fig. 2 shows an axisymmetric model for the analysis of a liquid bridge between a spherical object and a concave probe-tip, where $R$ is the radius of the object, $R_{\mathrm{p}}$ is the radius of the concavity, $D$ is the distance from the probe to the object, $\varphi$ is the filling angle of the object, $F$ is the attractive force acting on the object, and $V$ is the volume of the liquid bridge between two solids. The meniscus forms contact angles $\theta_{1}$ at the object and $\theta_{2}$ at the probe-tip. The profile of meniscus is expressed by the cylindrical coordinates $(X, Z)$. The value of $\varepsilon$ expresses the angle between the normal to the meniscus and the vertical axis. We make the following assumptions. (i) The influence of gravity is negligible and the profile of the liquid bridge follows Young-Laplace equation [9]; (ii) the dynamic flow of the liquid is negligible; (iii) the contact angles are determined by Young's equation [2].

Capillary force $F$ can be expressed as the sum of the pressure difference force and the surface tension force:

$$
F=-\Delta P \pi X_{1}^{2}+2 \pi \sigma X_{1} \sin \varepsilon_{1},
$$

where $\Delta P$ is Laplace pressure, i.e., the hydrostatic pressure difference between inside and outside the liquid, $\sigma$ is the surface tension, $X_{1}$ is the $X$-coordinate at the end-point of the profile on the object, and $\varepsilon_{1}$ is the $\varepsilon$-angle corresponding to the point $X_{1}$.

The Laplace pressure can be expressed by Young-Laplace equation, which relates the pressure difference to the local mean curvature $H$ and the surface tension $\sigma$;

$$
\Delta P=2 H \sigma .
$$

Since $\Delta P$ is hydrostatic, and thus, constant at any local point, the surface of the meniscus has the same mean curvature everywhere. As shown by Orr [9], the value of $H$ in equation (2) can be expressed by geometrical parameters as

$$
2 H=\frac{\mathrm{d}}{\mathrm{d} X}(\sin \varepsilon)+\frac{\sin \varepsilon}{X} .
$$


Since the left-hand side of this equation is constant, it can be solved as a two-pointboundary value problem, for which the boundary conditions are the $\varepsilon$-angle and $X$ coordinates of the menisci end on the solid surfaces. The $\varepsilon$-angles are determined by the slopes of the solid surfaces and the respective contact angles $\theta_{1}$ and $\theta_{2}$ (see fig. 2(a) and (b)). Fig. 2(b) shows three boundary states on the probe surface, which appears (1) on the concavity, (2) at the brim, and (3) on the flat surface. If one of the menisci end-point is on the concavity (see fig. 2(b-1)), the boundary conditions and corresponding $Z$ coordinate can be written as

$$
\left.\begin{array}{lll}
\varepsilon_{1}=\theta_{1}+\varphi, & X_{1}=R \sin \varphi, & Z_{1}=D+R(1-\cos \varphi), \\
\varepsilon_{2}=\pi-\theta_{2}+\phi_{1}, & X_{2}=R_{\mathrm{p}} \sin \phi_{1}, & Z_{2}=R_{\mathrm{p}}\left(1-\cos \phi_{1}\right),
\end{array}\right\}
$$

where $\phi_{1}$ is the filling angle of the concavity $\left(0 \leq \phi_{1} \leq \frac{\pi}{2}\right)$. When the menisci end reaches the brim of the concavity as fig. 2(b-2), i.e., in the case of $\phi_{1}=\frac{\pi}{2}$, the boundary condition on the probe surface is

$$
\varepsilon_{2}=\pi-\theta_{2}+\left(\frac{\pi}{2}-\phi_{2}\right), \quad X_{2}=R_{\mathrm{p}}, \quad Z_{2}=R_{\mathrm{p}},
$$

where $\phi_{2}$ is the angle changing at the brim of the concavity $\left(0 \leq \phi_{2} \leq \frac{\pi}{2}\right)$. In the case of fig. 2(b-3) for $\phi_{1}=\phi_{2}=\frac{\pi}{2}$, the boundary can be shown as

$$
\varepsilon_{2}=\pi-\theta_{2}, \quad X_{2}=R_{\mathrm{p}}+\phi_{3}, \quad Z_{2}=R_{\mathrm{p}}
$$

where $\phi_{3}$ is the $X$-displacement overflowed to the flat surface.

The boundary-value problem has the solution [9]. The meniscus profile $(X, Z)$, the distance $D$, the liquid volume $V$, and also the capillary force $F$ can be calculated from given four parameters; contact angles $\theta_{1}$ and $\theta_{2}$, the filling angle $\varphi$, and the parameter $\phi\left(\equiv \phi_{1}+\phi_{2}+\phi_{3}\right)$. If the volume $V$ is given in advance instead of the parameter $\phi$, the value of $\phi$ must be determined so that $V$ could be equal to the given value. Then, the relation between $D$ and $F$, which has the conservative liquid volume and given contact angles, can be plotted as a function of the filling angle $\varphi$. To generalize the following discussion, all the parameters are normalized as

$$
z=\frac{Z}{R}, \quad x=\frac{X}{R}, \quad d=\frac{D}{R}, \quad f=\frac{F}{\pi R \sigma}, \quad v=\frac{V}{R^{3}}, \quad r_{\mathrm{p}}=\frac{R_{\mathrm{p}}}{R} .
$$

Fig. 3 shows a relation between the normalized maximum capillary force and the normalized concavity radius for $v=0.10$. Both horizontal and vertical scale is logarithmic. Note that the variable of horizontal axis is not $r_{\mathrm{p}}$ but $r_{\mathrm{p}}-1$. Maximum value of the capillary force is the critical value of the object detachment from the concaved probe-tip. This figure suggests that as the radius of concavity approaches to the sphere, the maximum capillary force increases drastically, and also suggests that if the object and probe have smaller contact angles, capillary force becomes much larger than that for relatively large contact angles.

On the other hand, the capillary force should be reduced for placing manipulation. The solid lines in fig. 4 shows a relationship between the normalized 


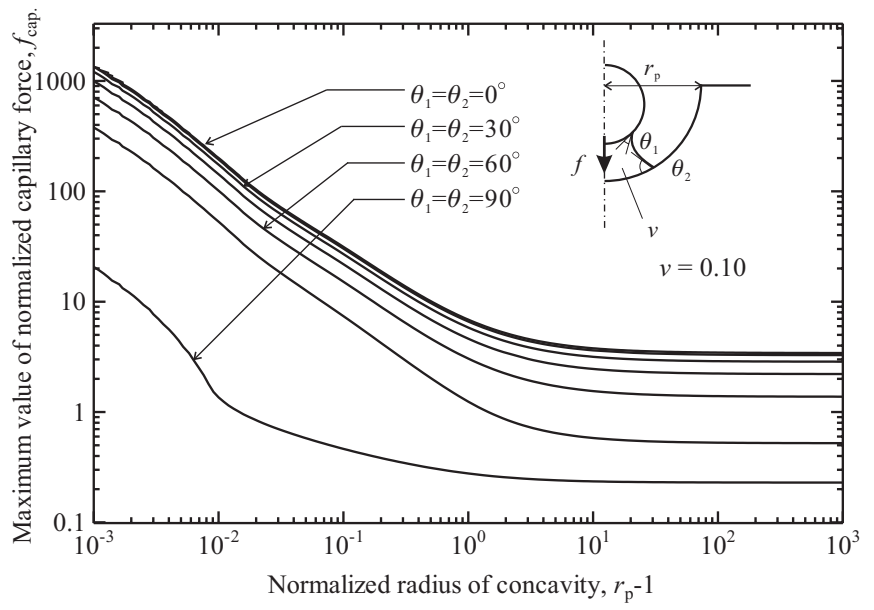

Figure 3: Relation between the normalized maximum capillary force $f_{\text {cap }}$ and the normalized radius of concave curvature $r_{\mathrm{p}}$ for the normalized liquid volume $v=0.10$.

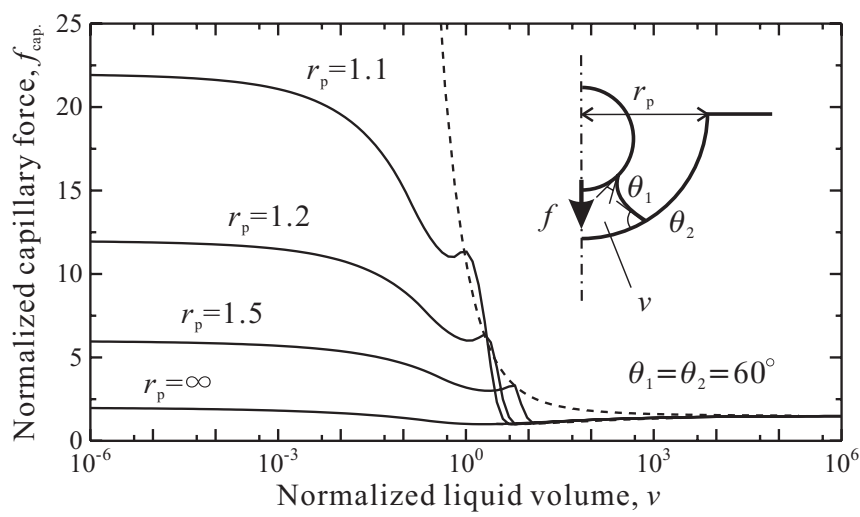

Figure 4: Relation between the normalized maximum capillary force $f_{\text {cap. }}$ and the normalized liquid volume $v$ for $\theta_{1}=\theta_{2}=60^{\circ}$.

maximum capillary force and the normalized liquid volume for $\theta_{1}=\theta_{2}=60^{\circ}$ and $r_{\mathrm{p}}=1.1,1.2,1.5$, and $\infty$. The infinite value of $r_{\mathrm{p}}$ means the sphere-plate model. As approaching $r_{\mathrm{p}}$ to 1 , the force difference by liquid volume regulation can be expanded. This means that the force control by the liquid volume is valid for reliable micromanipulation. 


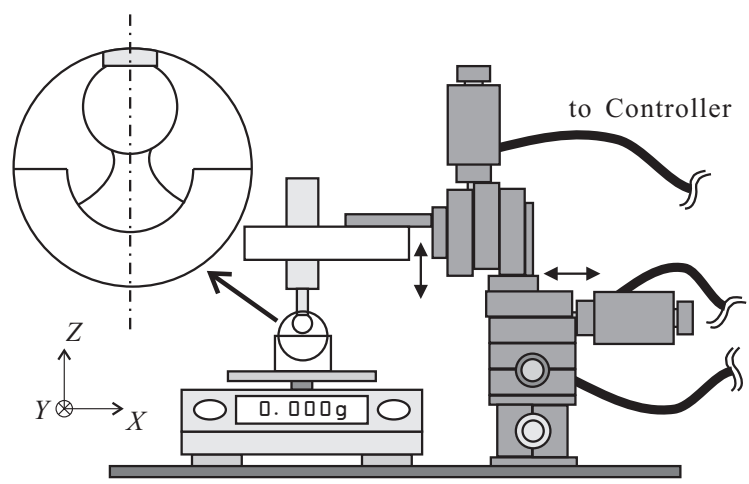

Figure 5: Schematic illustration of experimental system used. An electronic balance and a three-dimensional automated stage are fixed on a baseplate. An object functioning as a concave probe-tip is placed on the balance plate. The bigger circle shows the magnified cross-section of the smaller circle.

\section{Measurement of capillary force}

Fig. 5 shows the experimental system used for verification of numerical analysis. The experiment was performed in atmosphere. The order of the object size was determined according to the Bond Number $\left(\rho g R^{2} / \sigma\right.$, where $\rho$ is the density of the fluid and $g$ is the gravitational acceleration). Since the influence of gravity is negligible as long as the Bond Number is small enough, we adopted $R=$ $1.984-3.175 \times 10^{-3}[\mathrm{~m}]$ for the object radii corresponding to the bond number $\rho g R^{2} / \sigma=0.53-1.35$, which might shift the capillary force due to the gravity no more than $10 \%$. The liquid used was purified water with $\sigma=0.073[\mathrm{~N} / \mathrm{m}]$, which was refined through ion-exchange membrane process. A micro-pipette with a volume resolution of $2 \times 10^{-11}\left[\mathrm{~m}^{3}\right]$ was used to determine the volume of the liquid. An electronic balance (Sartorius, TE153S) with a resolution of $10^{-5}[\mathrm{~N}]$ was used to measure the force between the object and the probe-tip. Automated precise stages (Suruga Seiki, K701-20LMS) with a resolution of $5 \times 10^{-8}[\mathrm{~m}]$ were used to adjust the position of the object and the probe. Probes having concaved tips were fabricated with $R_{\mathrm{p}}=3.1 \times 10^{-3}[\mathrm{~m}]$ and $3.3 \times 10^{-3}$ [m] (of glass anpolytetrafluaroethylene: PTFE); with $R_{\mathrm{p}}=3.1 \times 10^{-3}$ [m] (of stainless steel). Spherical objects of several sizes were attached to steel rods. With the combination of the radii, the value of $r_{\mathrm{p}}$ can be set to 1.033-1.562. Contact angles for the materials were determined by observing the edge of a water-drop deposited on a plate using a video microscope: $50^{\circ}$ for glass, $75^{\circ}$ for stainless steal, and $85^{\circ}$ for PTFE.

Fig. 6 shows the measurement value of the capillary force $f$ as a function of the distance $d$ for the liquid volume $v=0.08$. The experimental value of the capillary force is obtained as square marks in an approaching process, and as triangle marks 


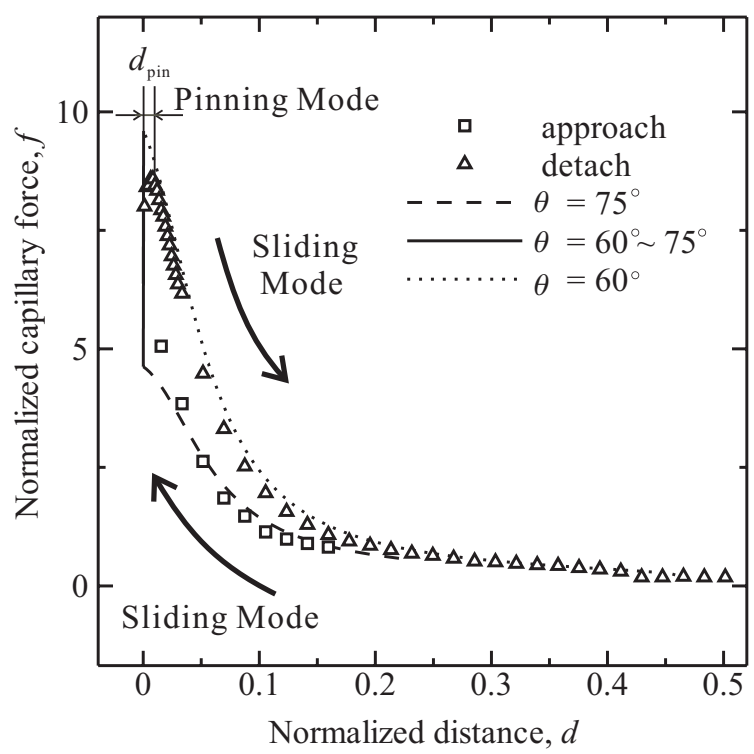

Figure 6: Relation between the normalized capillary force $f$ and the normalized distance $d$ for the normalized radius of concave curvature $r_{\mathrm{p}}=1.19$ and the normalized liquid volume $v=0.08$. Squares, and triangles denote data of force measurement for approaching and detaching process, respectively.

in a detaching process. Such hysteresis occurs due to the inequality of the contact angle between these processes. The broken line and the dotted line denote the theoretical value of the capillary force for the fixed contact angle $\theta_{1}=\theta_{2}=60^{\circ}$ and $75^{\circ}$ (Sliding Mode according to Pitois [10]). The solid line denotes the value of the force for the contact angle transition from $60^{\circ}$ to $75^{\circ}$ (Pinning Mode according to Pitois [10]). In this case, because of contact angle hysteresis, the observed capillary force never achieves the maximum value of the theoretical prediction.

The relations between the normalized maximum capillary force $f_{c} a p$. and the normalized radius of concaved curvature $r_{\mathrm{p}}$ for a given normalized liquid volume $v=0.08$ are shown in fig. 7. Lines denote the values estimated from the numerical analysis for contact angles $\theta_{1}=\theta_{2}=50^{\circ}, 75^{\circ}$, and $85^{\circ}$. Circle, square, and triangle marks denote the values actually measured in the experiment. Both horizontal and vertical scales are logarithmic. Note that the variable of the horizontal axis is $r_{\mathrm{p}}-1$ instead of $r_{\mathrm{p}}$. The experimental results are in good agreement with the theoretical predictions for all three materials. The force $f_{\text {cap. }}$. drastically increases as $r_{\mathrm{p}}$ approaches 1 ,which suggests that a probe with the concave dimension closer to the convex dimension of the object can generate much larger capillary force. Probes made of the material with small contact angle generate much larger capillary force. For $r_{\mathrm{p}}$ closer to 1, the larger differences are 
found between the measured values and the theoretical predictions. We presume the reason for this is that the error of the positional adjustment has relatively larger influence on the generated force as the concave radius approaches the convex radius of the object.

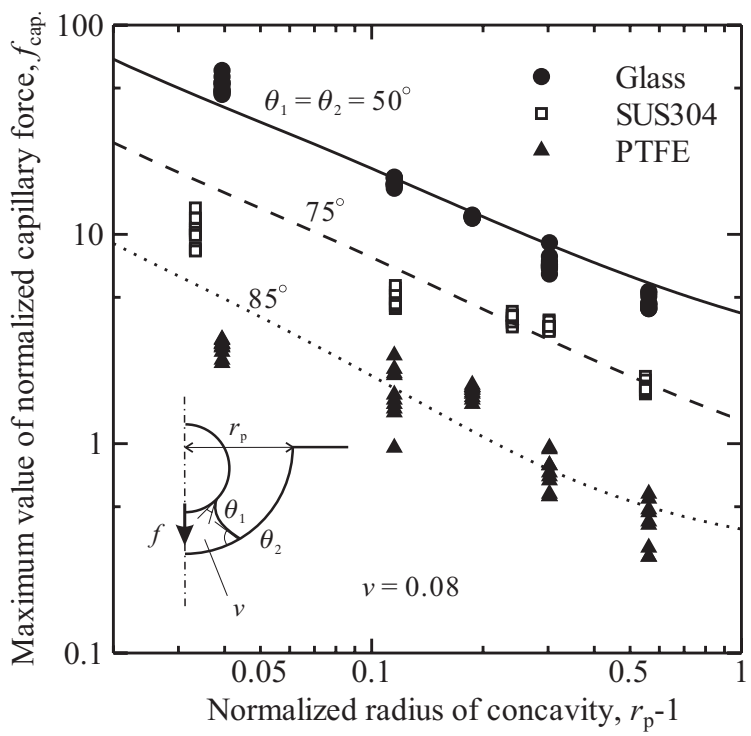

Figure 7: Relation between the normalized maximum capillary force $f_{\text {cap }}$ and the normalized radius of concave curvature $r_{\mathrm{p}}$ for the normalized liquid volume $v=0.08$. Circles, squares, and triangles denote data of force measurement for glass, stainless steel, and polytetrafluoroethylene (PTFE), respectively.

As shown in fig. 8, magnified capillary force can be controlled by the regulation of liquid volume. Circle, triangle, and square marks are expressing the experimental values. These are in good agreement with theoretical predictions for $v>0.1$. In the case of $v<0.1$, the experimental value of capillary force and the calculation considering contact angle hysteresis (broken lines) are almost the same. The broken lines are calculated by neglecting the capillary force during the pinning mode $\left(0 \leq d \leq d_{\text {pin }}\right)$. Assuming the pinning mode distance $d_{\text {pin }}$ is constant, the capillary force is reduced with decreasing liquid volume. This means that too small supply of liquid causes less capillary force generated. In order to realize efficient and reliable manipulation, the normalized liquid volume should be controlled from 0.1 to 10 . 


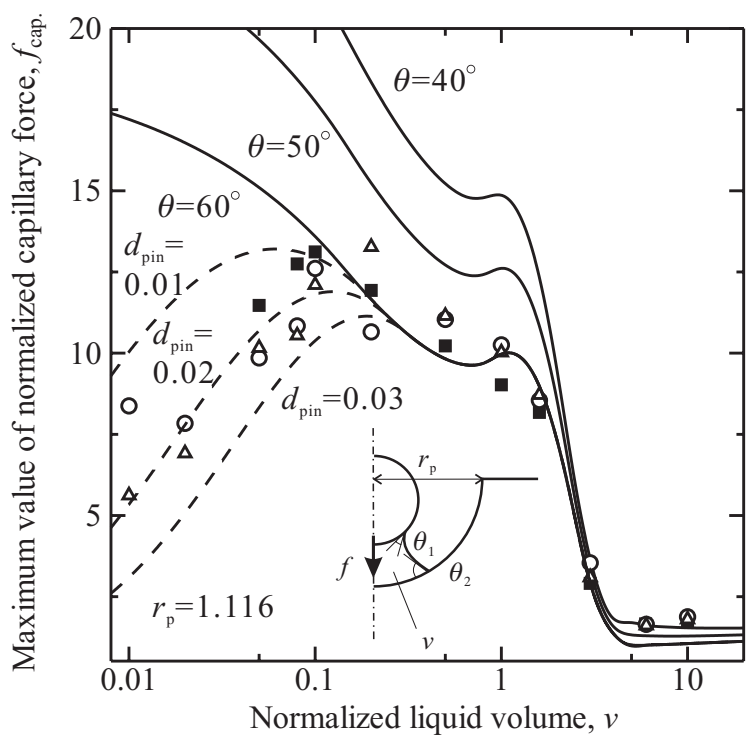

Figure 8: Relation between the normalized maximum capillary force $f_{\text {cap. }}$ and the normalized liquid volume $v$ for glass specimen and $r_{\mathrm{p}}=1.116$.

\section{Conclusion}

Through both analysis and measurement of capillary force, this study clarifies the most important factors for reliable capillary micromanipulation by concave probe, i.e. the material wettability, the concave shape and dimension, and the amount of liquid supply. The shape of the probe-tip can be designed as shown in fig. 1 so that the range of the capillary force can be extended due to the change of the apparent contact angle for a given liquid volume. The more wettable a material is, the greater capillary force it can generate. The magnified capillary force can be reduced/controlled by liquid volume regulation. This suggests that micromanipulation by capillary force has a great potential for a wide range of applications. In the capillary force measurement, the contact angle hysteresis can be observed, and we presume that it reduces the maximum value of the capillary force in case of relatively small liquid volume. For actual manipulation, a mechanism that is able to supply proper amounts of liquid needs to be developed.

\section{References}

[1] K.L. Johnson, K. Kendall, and A.D. Roberts, Surface energy and the contact of elastic solids. Proc. R. Soc. Lond. A., 324, pp. 301-313, 1971

[2] Jacob N. Israelachvili, Intermolecular and Surface Forces. Academic Press, New York, pp. 301-322, 1985

[3] Shigeki Saito, Hideki T. Miyazaki, and Tomomasa Sato, Micro-object Pick 
and Place Operation under SEM based on Micro-physics. J. Robotics and Mechatronics, 14, pp. 227-237, 2002

[4] Shigeki Saito, Hideki T.Miyazaki, Tomomasa Sato, and Kunio Takahashi, Kinematics of mechanical and adhesional micromanipulation under a scanning electron microscope. J. Applied Physics, 92(9), pp. 5140-5149, 2002

[5] Kunio Takahashi, Hideaki Kajihara, Masataka Urago, and Shigeki Saito, Voltage required to detach an adhered particle by Coulomb interaction for micromanipulation. J. Applied Physics, 90(1), pp. 432-437, 2001

[6] Shigeki Saito, Hideo Himeno, and Kunio Takahashi, Electrostatic detachment of an adhering particle from a micromanipulated probe. $J$. Applied Physics, 93(4), pp. 2219-2224, 2003

[7] Tamio Tanikawa, Yoshiyuki Hashimoto, and Tatsuo Arai, Micro Drops for Adhesive Bonding of Micro Assemblies and Making a 3-D Structure 'Micro Scarecrow'. Proceedings of the IEEE/RSJ Intl. Conference on Intelligent Robotics and Systems, pp. 776-781, 1998

[8] Kenichi J. Obata, Tomoyuki Motokado, Shigeki Saito, and Kunio Takahashi J. Fluid Mech., 498 pp. 113-121, 2004

[9] F.M. Orr, L.E. Scriven, and A.P. Rivas, Pendular rings between solids: meniscus properties and capillary force. J. Fluid Mech., 67(4), pp. 723-742, 1975

[10] Oliver Pitois and Xavier Chateau, Small Particle at a Fluid Interface: Effect of Contact Angle Hysteresis on Force and Work of Detachment, Langmuir, 18, pp. 9751-9756, 2002 